\title{
Single and multi-area multi-fuel economic dispatch using a fuzzified squirrel search algorithm
}

\author{
V. Ponnuvel Sakthivel ${ }^{1 *}$ and P. Duraisamy Sathya ${ }^{2}$
}

\begin{abstract}
Multi-Area Multi-Fuel Economic Dispatch (MAMFED) aims to allocate the best generation schedule in each area and to offer the best power transfers between different areas by minimizing the objective functions among the available fuel alternatives for each unit while satisfying various constraints in power systems. In this paper, a Fuzzified Squirrel Search Algorithm (FSSA) algorithm is proposed to solve the single-area multi-fuel economic dispatch (SAMFED) and MAMFED problems. Squirrel Search Algorithm (SSA) mimics the foraging behavior of squirrels based on the dynamic jumping and gliding strategies. In the SSA approach, predator presence behavior and a seasonal monitoring condition are employed to increase the search ability of the algorithm, and to balance the exploitation and exploration. The suggested approach considers the line losses, valve point loading impacts, multi-fuel alternatives, and tie-line limits of the power system. Because of the contradicting nature of fuel cost and pollutant emission objectives, weighted sum approach and price penalty factor are used to transfer the bi-objective function into a single objective function. Furthermore, a fuzzy decision strategy is introduced to find one of the Pareto optimal fronts as the best compromised solution. The feasibility of the FSSA is tested on a three-area test system for both the SAMFED and MAMFED problems. The results of FSSA approach are compared with other heuristic approaches in the literature. Multi-objective performance indicators such as generational distance, spacing metric and ratio of non-dominated individuals are evaluated to validate the effectiveness of FSSA. The results divulge that the FSSA is a promising approach to solve the SAMFED and MAMFED problems while providing a better compromise solution in comparison with other heuristic approaches.
\end{abstract}

Keywords: Fuzzy set theory, Heuristic optimization, Multi-area economic dispatch, Pareto-optimal front, Squirrel search algorithm, Tie-line constraint

\section{Introduction}

The goal of Multi-Area Multi-Fuel Economic Dispatch (MAMFED) is to decide the power delivered by every generator in all the areas and the power flow between the areas in order to lessen the total production cost and pollutants outflows of the interconnected power system considering multi-fuel alternatives of each generating unit. The total load power is shared among the various

\footnotetext{
* Correspondence: vpsarvesh2013@gmail.com

'Department of Electrical and Electronics Engineering, Government College of Engineering, Dharmapuri, Tamilnadu 636 704, India

Full list of author information is available at the end of the article
}

areas to decrease the fuel cost as well as the pollutant emission, while the power scheduling between the zones must consider the power balance, generator and tie-line limits. Thus, the MAMFEED problem which has received considerable attention in recent years, is addressed as a large-scale highly non-linear multiobjective optimization problem.

\subsection{Literature review}

Over the years, various classical multi-area power generation scheduling methods have been proposed. An efficient approach is applied in [1] for unit 
commitment and Economic Dispatch (ED) problems with area import/export constraints. The proposed approach is tested on the Texas utilities and Texas municipal power pool systems. In [2], it suggests the use of the Dantzig-Wolfe decomposition principle regarding the revised simplex method and a fastdecoupled power flow algorithm for constrained Multi-Area ED (MAED) of power systems. A model of large-scale multi-area power generation system is developed in [3] and a rule-based heuristic strategy is used to improve the generation schedule for every zone. Reference [4] proposes a decomposition approach which upgrades the scheduling process and accelerates the execution of a large-scale multi-area generation system in a real-time application. The MAED problem is expressed as a capacitated nonlinear network flow problem in [5] and is solved through an Incremental Network Flow Programming (INFP) approach. The results show that the INFP approach is fast, robust, and extendable to large-scale systems. An Improved Hopfield Neural Network (IHNN) is presented to solve MAED problems with transmission capacity constraints which shows that IHNN achieves efficient and accurate solutions for the two-area power system [6]. In [7], a Hopfield Lagrange network is proposed to solve economic emission dispatch (EED) problem with multiple fuel alternatives (MFAs), while the best compromise from the set of obtained solutions is found and compared with that from the Lamda-Iteration method.

Despite the fact that these traditional strategies can give a strong ideal solution, they experience the disadvantages of having non-smooth and non-convex objective functions. Thus, these strategies do not offer reasonable possibilities for dealing with the MAED problem when prohibited operating zones (POZs), valve point loading (VPL), and MFAs are considered [8]. To adapt to these challenges, numerous meta-heuristic methodologies have been used to take care of enhancement issues with complicated objectives [9]. The results obtained by evolutionary approaches for complex optimizing problems are good, which means these approaches offer a reasonable possibility for solving MAED problems.

In recent years, swarm intelligence algorithms have been broadly used to overcome the computational unpredictability issues in the MAED problem. Reference [10] proposes an efficient technique for MAED problems using an Evolutionary Programming (EP) approach, while the performance of the various evolutionary algorithms, including the Real-Coded Genetic Algorithm (RCGA), Particle Swarm Optimization (PSO), Differential Evolution (DE), and the Covariance Matrix Adapted Evolution Strategy (CMAES), on MAED problems with
Karush-Kuhn-Tucker optimality conditions are examined [11]. The simulation results revealed that the CMAES algorithm offers the best results and the Nelder-Mead simplex method offers an optimal solution in the shortest time among the different algorithms considered. The performance of different DE strategies enhanced with time-varying mutation is investigated and analyzed to solve the reserve-constrained MAED problem [12]. The time varying DE approach is found to be capable of determining better solutions than PSO with time-varying acceleration coefficients. In addition, the $\mathrm{DE}$ variant approaches are able to find the global best solutions for large-scale systems, while basic DE suffers from premature convergence.

A computationally efficient fuzzified PSO algorithm is introduced in [13] to solve the securityconstrained MAED problem of an interconnected power system. The inertia weight of the standard PSO is made adaptive by using fuzzy logic strategy to improve the convergence speed and avoid premature convergence. Artificial bee colony $(\mathrm{ABC})$ optimization is proposed to solve a MAED problem with tie-line constraints, transmission losses, multiple fuels, and valve point effects [14]. Evolutionary approaches such as DE, EP, and RCGA are applied to analyze the efficiency of the $A B C$ approach. The simulation results show that the $\mathrm{ABC}$ approach converges to a better solution than other comparable approaches. Teaching learning-based optimization (TLBO) has been applied to solve the MAED issue [15]. The approach is tested on three different systems and the results reveal that the presented approach has the ability to provide better solutions and exhibits greater robustness than DE, EP, and RCGA. The different MAED models are solved by employing Fast Convergence Evolutionary Programming (FCEP) in [16] which uses Gaussian and Cauchy mutations to improve the convergence speed and solution quality. The Hybrid Cuckoo Search Algorithm (HCSA) which consolidates the cuckoo search algorithm and TLBO to improve the performance of optimization process, is developed to solve the MAED problem [17]. In [18], a hybrid DE-PSO technique is used to address the MAED, reserveconstrained MAELD, and reserve-constrained multiarea ecological/economic dispatch problems. Sum Differential Evolution with Particle Swarm Optimizer (SDEPSO) is used along with the local (Pbest) optimal value in a DE crossover operator. The simulation results show that the hybrid algorithm achieves a good balance between global search ability and provision of better convergence and solution quality.

An Improved Grasshopper Optimization Algorithm (IGOA) is introduced to deal with the MAED 
problem [19], in which a chaos mechanism is adopted to handle the premature convergence of the grasshopper optimization algorithm. The simulation results demonstrate that IGOA converges better than the other approaches. Backtracking Search Algorithm (BSA) is proposed to solve ED problems considering VPL effects, POZs, and MFAs [20], while the MAED problem is solved by combining Jaya and TLBO approaches to simultaneously solve both non-smooth and non-convex characteristics [21]. The Jaya algorithm consistently attempts to find the best solution and avoids the worst solution, and thus is endeavoured to be successful by arriving at the best solution. A Parallel MAED (PMAED) is developed to maintain the independency and transfer the required power in each area [22], which is solved with convex and non-convex fuel cost functions of different complexities. A Newton Method based Distributed Algorithm (NMDA) and Evolutionary PSO (EPSO) algorithm are employed to solve Single-Area Economic Dispatch (SAED) and MAED problems [23, 24], where the virtual SAED formulation, backtracking line search algorithm, and average consensus theory are embedded in the NMDA approach. In the EPSO algorithm, the GA operators such as mutation, crossover, and selection are employed to enable the search process. A new heuristic approach, the Coulomb's and Franklin's laws-based optimization (CFLBO) algorithm is developed to solve the non-convex economic and emission dispatch problem [25]. These improved and hybrid forms of heuristic approaches involve complicated computation owing to the use of many control parameters. Thus, an efficient algorithm to solve MAED problems to ascertain the optimum dispatch solutions needs be developed.

\subsection{Contributions}

Recently, a new meta-heuristic algorithm, named as Squirrel Search Algorithm (SSA) was proposed in [26]. SSA models the foraging activities of individual squirrels, while each squirrel modifies its position using four processes, namely:

(1) distributing the population,

(2) dynamic foraging behavior,

(3) seasonal adapting intelligence, and.

(4) random repositioning of individuals at the end of winter season.

The unique features of SSA are as follows:

- The gliding constant is used in the location update of squirrels which provides suitable steadiness between exploration and exploitation.
- The predator presence behavior is employed to abruptly change the squirrel locations which enhances the exploration ability of the algorithm.

- A seasonal monitoring condition is used to prevent the suggested algorithm from being trapped in local optimal solutions.

- Levy distribution is used to find new solutions far away from the current best solution which improves the global exploration ability of the algorithm.

These features make SSA capable of overcoming the normal drawbacks of other algorithms such as premature convergence, inadequate ability to find nearby extreme points and absence of efficient constraints handling mechanism. The advantages of the SSA approach are less execution time, ability to solve different complex optimization problems and high capacity in obtaining global optimum solutions. Thus, the SSA approach is able to provide solutions of better quality than the existing heuristic approaches.

The main contributions of this paper are briefly summarized as follows:

- For the first time, the application of the SSA approach for solving the Single-Area Multi-Fuel ED (SAMFED) and MAMFED problems in power system is reported.

- A FSSA approach to solve the SAMFED and MAMF ED problems is introduced. The proposed algorithm involves the SSA approach, weighted sum approach and fuzzy decision strategy. The weighted sum approach is used to transfer the bi-objective functions such as fuel cost and pollutant emission into a single objective function, while the fuzzy decision strategy is used to provide the best compromised solution from the non-dominated solution set. The FSSA approach successfully solves the SAMFED problem such that the fuel cost and pollutant emission are simultaneously minimized while fulfilling the power balance and generation limits.

- The SSA approach is successfully applied to solve the MFMAED such that the total fuel cost is minimized while fulfilling the system and area constraints.

- The proposed algorithm is applied to a three-area 10-unit system to demonstrate its effectiveness in solving the SAMFED and MAMFED problems. The obtained results are compared with different stateof-the-art heuristic approaches.

The structure of this paper is as follows. Section 2 presents the details of the SAMFED and MAMFED models, while the recommended FSSA approach is presented in Section 3. The implementation of the FSSA approach 
for the ED problems is discussed in Section 4 and in Section 5, the numerical results are discussed and the adequacy of the proposed FSSA approach is demonstrated. Finally, Section 6 concludes the paper.

\section{Problem formulation of multi-fuel economic dispatch}

2.1 SAMFED

\subsubsection{Fuel cost objective function}

The goal of the ED problem is to limit the total expense of thermal units as:

$$
\text { Minimize } F=\sum_{i=1}^{n g} F_{i}\left(P_{i}\right)
$$

In the ED problem, the fuel cost of each generating unit is communicated as a quadratic function of its capacity yield. As the generating units utilize different fuel choices to create power and consider the valve point impacts, the fuel cost function is expressed in (6).

The multi-fuel cost curves with and without VPL impacts are shown in Fig. 1.

\subsubsection{Emission objective function}

The objective of the emission dispatch (EmD) problem is to lessen the hazardous emissions as:

$$
\text { Minimize } E=\sum_{i=1}^{n g} E_{i}\left(P_{i}\right)
$$

The emission objective function is similar to the fuel cost function while it deals with all discharge types by generation units. The model for emanation function with MFA is introduced in (7).

\subsubsection{Economic and emission dispatch (EED) function}

The EED problem can be considered as bi-target work with fuel cost and discharge as equalling objectives. This

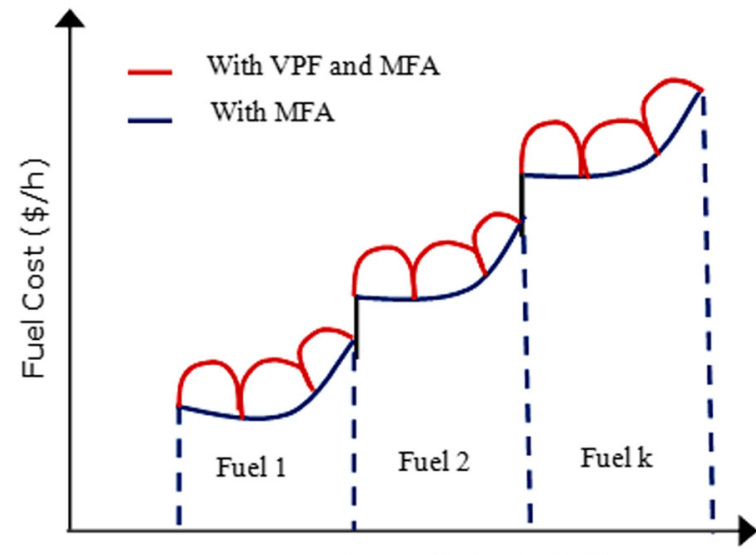

Power Output (MW)

Fig. 1 Multi-fuel cost curve bi-target function can be merged into a solitary target function as:

$$
\text { Minimize } F_{E E D}=w \times F+h \times(1-w) \times E
$$

where $\mathrm{w}$ is a primary function of rand $[0,1]$ which bargains the fuel cost and emanation objectives. So the above condition becomes ED target function when $\mathrm{w}=1$ and becomes EmD target when $\mathrm{w}=0$.

\subsubsection{Power balance constraint}

The total power generated from a set of committed units must fulfil the total load demand, i.e.:

$$
\sum_{i=1}^{n g} P_{i}=P_{D}
$$

\subsubsection{Generator capacity limits}

The real output power of thermal units need to be in their minimum and maximum limits as:

$$
P_{i, \min } \leq P_{i} \leq P_{i, \max }
$$

$$
F i\left(P_{i}\right)=\left\{\begin{array}{c}
\text { Fuel type } 1 ; a_{i 1}+b_{i 1} P_{i}+c_{i 1} P_{i}^{2}+\left|e_{i 1} \times \sin \left(f_{i 1} \times\left(P_{i, \min }-P_{i}\right)\right)\right| ; P_{i, \min } \leq P_{i} \leq P_{i 1} \\
\text { Fuel type } 2 ; a_{i 2}+b_{i 2} P_{i 2}+c_{i 2} P_{i}^{2}+\left|e_{i 2} \times \sin \left(f_{i 2} \times\left(P_{i, \text { min }}-P_{i}\right)\right)\right| ; P_{i 1}<P_{i} \leq P_{i 2} \\
\cdots \\
\text { Fuel type } k ; a_{i k}+b_{i k} P_{i}+c_{i k} P_{i}^{2}+\left|e_{i k} \times \sin \left(f_{i k} \times\left(P_{i, \text { min }}-P_{i}\right)\right)\right| ; P_{i, k-1}<P_{i} \leq P_{i, \text { max }}
\end{array}\right.
$$

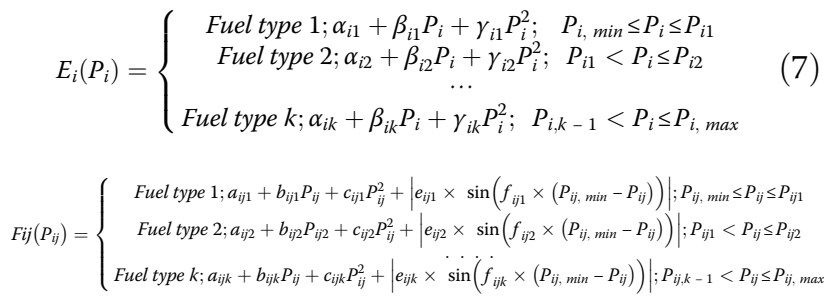

\subsection{MAMFED}

\subsubsection{Fuel cost objective function}

The aim of the MAMFED problem is to find the amount of power that can be efficiently generated in one area and transferred to another area, and to determine the economic fuel choice for each unit. Since generators are provided with multi-fuel sources, every generator needs to be defined with a few piecewise quadratic capacities superimposed by sine terms mirroring the impact of changes in the type of fuel while the generator must determine the most conservative fuel to consume. The fuel cost function with VPL and MFA impacts [14] is defined in (8). 


\subsubsection{Power balance constraint}

The total power generated by a set of accessible units must satisfy the total load demand, tie-line power flow, and transmission losses [9] as:

$$
\sum_{j=1}^{M_{i}} P_{i j}=P_{D i}+P_{L i}+\sum_{z, z \neq i} T_{i z}
$$

where $i=1,2, \ldots n_{g}, j=1,2, \ldots M_{i}$.

\subsubsection{Generator capacity limits}

The real output power of the thermal units should be in the minimum and maximum limits as [9]:

$$
P_{i j, \min } \leq P_{i j} \leq P_{i j, \max }
$$

\subsubsection{Tie-line limit}

Because of security basis, power transmitted through various lines must not surpass their cutoff points [11]. The power transfer requirement between two regions is characterized by:

$$
-T_{i z, \min } \leq T_{i z} \leq T_{i z, \max }
$$

\section{Brief overview of the FSSA approach}

\subsection{SSA}

The hunt procedure starts when flying squirrels begin scavenging [26]. During fall, squirrels look for nourishment assets by skimming from one tree to the next. At the same time, they change their areas and investigate various regions of woods. As the climatic conditions are sufficiently hot, they can meet their every day vitality needs more rapidly on the eating routine of oak seeds accessible in bounty and thus, they devour oak seeds quickly after discovering them. Subsequent to satisfying their day by day vitality prerequisite, squirrels scan for ideal nourishment hotspot for winter (hickory nuts). Capacity of hickory nuts will help them in keeping up their vitality prerequisites in harsh climate, decrease the expensive searching excursions and increase the likelihood of endurance.

During winter, lost leaves spread in deciduous woodlands result an expanded danger of predation and thus, squirrels become less dynamic but still remain active. Toward the finish of winter season, squirrels again become dynamic. This is monotonous procedure and structures the establishment of SSA. The SSA approach refreshes the places of squirrels as indicated by the ebb and flow season, the sort of squirrels and if chasers showing up.

\subsubsection{Instate the population}

Assuming the number of squirrels is $N$, and the upper and lower limits of the pursuit space are $X_{U}$ and $X_{L}$, the $N$ squirrels are arbitrarily created as:

$$
X_{i}=X_{L}+\operatorname{rand}(1, D) \times\left(X_{U}-X_{L}\right)
$$

where $X_{i}$ indicates the $i^{\text {th }}$ squirrel, $(i=1: N)$, rand () is an random number in the range of 0 and 1 , and $D$ is the measurement of the issue.

\subsubsection{Group the population}

SSA requires that there is only a single squirrel at each tree, so for $N$ squirrels, there are $N$ trees in the woods. Among the $N$ trees, there is one hickory tree and $N_{a}$ oak seed trees, while the rests are typical trees having no nourishment. The hickory tree is the best nourishment asset for the squirrels while the oak seed trees come the second. Positioning the fitness estimations of the populace in rising request, the squirrels are separated into three kinds:

- Squirrels situated at hickory tree $\left(W_{h}\right)$;

- Squirrels situated at oak seed trees $\left(W_{a}\right)$;

- Squirrels situated at ordinary trees $\left(W_{n}\right)$.

\subsubsection{Refresh the location of squirrels}

The squirrels refresh their situations by skimming to the hickory tree or oak seed trees as follows:

$$
\begin{aligned}
& X_{i}^{t+1}=\left\{\begin{array}{cc}
X_{i}^{t}+d_{g} G_{c}\left(X_{a i}^{t}-X_{i}^{t}\right) & \text { if } r_{2} \geq P_{d p} \\
\text { Random location } & \text { otherwise }
\end{array}\right. \\
& X_{i}^{t+1}=\left\{\begin{array}{cc}
x_{i}^{t}+d_{g} G_{c}\left(X_{h}^{t}-X_{i}^{t}\right) & \text { if } r_{3} \geq P_{d p} \\
\text { Random location, } & \text { otherwise }
\end{array}\right.
\end{aligned}
$$

$P_{d p}$ is esteemed at 0.1 and indicates the chaser likelihood. In the event that $r>P_{d p}$, no chaser shows up, and the squirrels coast in the backwoods to discover the nourishment and are protected. If $r<P_{d p}$, the chasers show up, and the squirrels are compelled to limit the extent of exercises and are imperiled, and their locations are migrated arbitrarily. $d_{g}$ is the skimming separation that can be determined by:

$$
d_{g}=\frac{h_{g}}{\tan (\phi)}
$$

where $h_{g}$ is the constant estimated $8, \tan (\phi)$ indicates the coasting point that can be determined by:

$$
\tan (\phi)=\frac{D}{L}
$$

The drag power and lift power can be estimated as: 


$$
\begin{aligned}
& D=\frac{1}{2 \rho V^{2} S C_{D}} \\
& L=\frac{1}{2 \rho V^{2} S C_{L}}
\end{aligned}
$$

\subsubsection{Occasional changeover verdict and arbitrary refreshing}

Toward the start of every generation, SSA necessitates that the entire populace is in winter, which implies that the locations of all squirrels are updated by (11) and (12). At the point when the squirrels are refreshed, regardless the season, change is decided by the following formulae:

$$
\begin{aligned}
& S_{c}^{t}=\sqrt{\sum_{k=1}^{d}\left(X_{a i, k}^{t}-X_{h, k}^{t}\right)^{2}} i=1,2, . ., N_{a} \\
& S_{\text {min }}=\frac{10 e^{-6}}{365^{t /\left(t_{\max } / 2.5\right)}}
\end{aligned}
$$

If $S_{t c}<S_{\text {min }}$, winter is finished and the season goes to summer, otherwise the season is unaltered. At the point when the season goes to summer, the squirrels who float to $W_{h}$ remain at the refreshed area, while the squirrels skimming to $W_{a}$ and not meeting with chasers move their situations as follows:

$$
X_{\text {inew }}^{t+1}=X_{L}+L e^{\prime} v y(x) \times\left(X_{U}-X_{L}\right)
$$

Le'vy is the arbitrary walk model whose progression complies with the Le'vy appropriation and can be determined by:

$$
\operatorname{Le}^{\prime} v y(x)=0.01 \times \frac{\alpha \times r_{a}}{\left|r_{b}\right|^{\frac{1}{\beta}}}
$$

where $\alpha$ is determined as:

$$
\alpha=\left[\frac{\Gamma(1+\beta) \times \sin \left(\frac{\pi \beta}{2}\right)}{\Gamma\left(\frac{1+\beta}{2}\right) \times \beta \times 2^{\left(\frac{\beta-1}{2}\right)}}\right]^{\frac{1}{\beta}}
$$

\subsection{Fuzzy decision strategy}

The objective functions, viz. economic and emission dispatch shall be used in parallel in the multi-objective EELD problem. However, this makes the comparison of the two solutions difficult. Neither solution vector $X_{1}$ nor $X_{2}$ can be superior to each other if they are Pareto optimal, because if superior result is obtained from $X_{1}$ for an objective, $X_{2}$ would offer improved performance for another objective. Obtaining the best solution from multiple non-dominated solutions is challenging in multi-objective EELD problem, though it is always possible to collate these outcomes and obtain the best compromised solution. For achieving this, one has to use the proper mechanism to combine both objectives and ensure that it conforms to the target and preference of the decision maker.

Researchers commonly use fuzzy set theory to arrive at the best solution amongst many uncontrolled solutions. It is implausible to achieve both least fuel cost along with least emission as they are contrary to each other. But it is feasible to build a dispatch option that can optimize both. Fuzzy membership functions assign Degree of agreement (DA) to each objective, and merit of the objective is reflected by DA in a linear scale of 0 -1 (worst to best). $F_{j}$ is a solution in the Pareto-optimal set in the $j^{\text {th }}$ objective function and is defined by a membership function as:

$$
\mu\left(F_{j}\right)=\left\{\begin{array}{cc}
1 & \text { if } F_{j} \leq F_{j}^{\min } \\
\frac{F_{j}^{\max }-F_{j}}{F_{j}^{\max }-F_{j}^{\min }} & \text { if } F_{j}^{\min } \leq F_{j} \leq F_{j}^{\max } \\
0 & \text { if } F_{j} \geq F_{j}^{\max }
\end{array}\right.
$$

For each non-dominated solution, the normalized membership function $\mu_{D}^{k}$ can be calculated as:

$$
\mu_{D}^{k}=\frac{\sum_{i=1}^{2} \mu\left(F_{i}^{k}\right)}{\sum_{k=1}^{M} \sum_{i=1}^{2} \mu\left(F_{i}^{k}\right)}
$$

The solution that contains the maximum of $\mu_{\mathrm{D}}^{\mathrm{k}} \mu_{D}^{k}$ based on cardinal priority ranking is the best compromised solution, i.e.:

$$
\operatorname{Max}\left\{\mu_{D}^{k}: k=1,2, . ., M\right\}
$$

\section{Implementation of FSSA for SAMFED and MAMF ED problems}

The steps of the solution for MFED problem using FSSA are as follows.

Step 1: Randomly generate the generation values between lower and upper power outputs of each generating unit in all solutions.

Step 2: Evaluate the objective values of all the squirrels using (3).

Step 3: Sort the objective values of each squirrel's position in ascending order.

Step 4: Declare the flying squirrel with low fitness value as on the hickory nut tree (optimal food source), the next three best flying squirrels on the acorn tree (normal food source) and the rest of the squirrels on the normal trees (no food source).

Step 5: Update the positions of squirrels located on the oak seed and normal trees using (12), (13) and (14). 
Step 6: Randomly relocate the positions of some squirrels when seasonal monitoring condition is satisfied.

Step 7: Repeat steps 2 to 6 until stopping criterion is met.

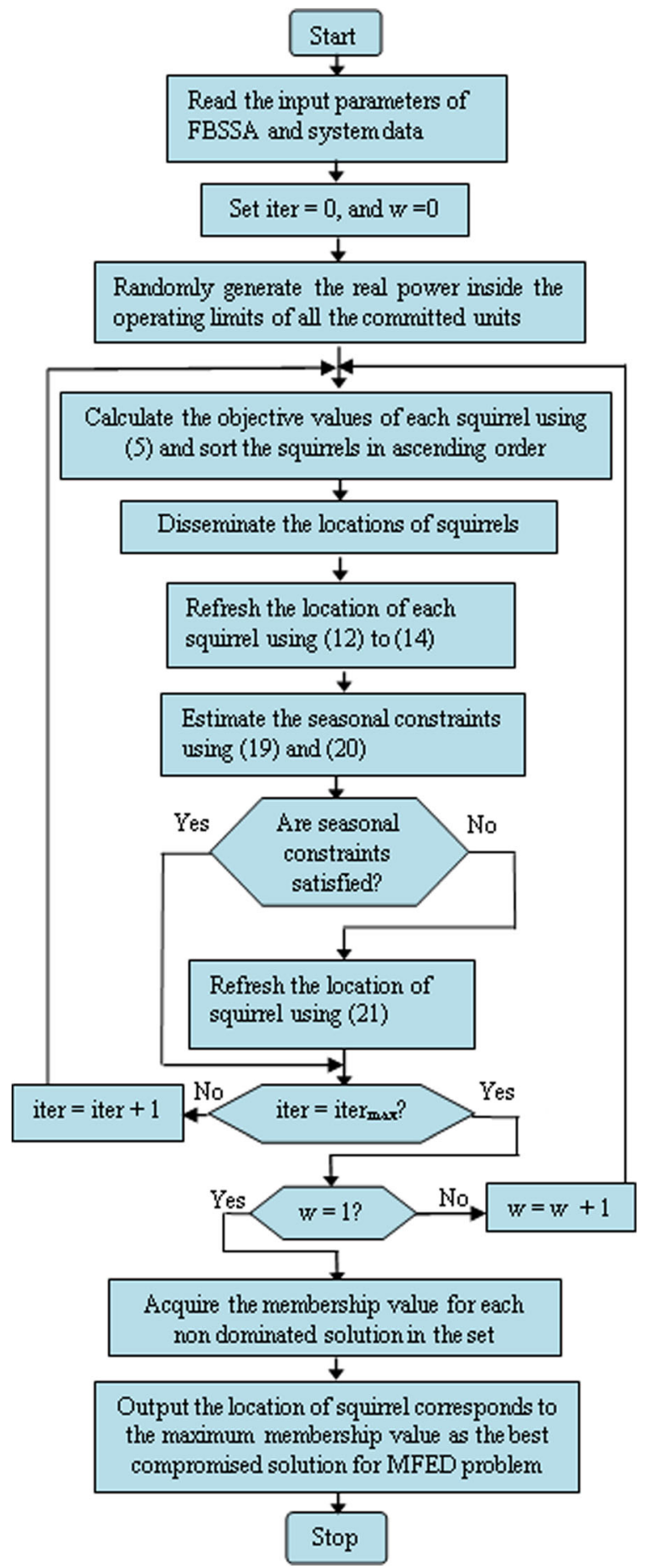

Fig. 2 Flow chart of FSSA algorithm for solving the MFED problem
Table 1 Optimum FSSA parameters

\begin{tabular}{ll}
\hline Parameter & Value \\
\hline Number of hickory tree & 1 \\
Number of acorn trees $\left(N_{a}\right)$ & 3 \\
Predator presence probability $\left(P_{d p}\right)$ & 0.1 \\
Gliding constant $(G c)$ & 1.9 \\
Maximum iteration number & 200 \\
Population size $\left(N_{p}\right)$ (Test system 2) & 20 \\
Density of air $(\rho)$ & $1.204 \mathrm{kgm}^{-3}$ \\
Speed $(V)$ & $5.25 \mathrm{~ms}^{-1}$ \\
Surface area of body $(S)$ & $154 \mathrm{~cm}^{2}$ \\
Drag coefficient $\left(C_{D}\right)$ & 0.6 \\
Lift coefficients $\left(C_{L}\right)$ & $0.675 \leq C_{L} \leq 1.5$ \\
\hline
\end{tabular}

Step 8: Increment the weight factor in step of 0.1 and repeat steps 2 to 6 , until the weight factor reaches unity.

The membership value for each non-dominated solution sets which are acquired for different weight factors using (24) is determined, and the position of squirrel on hickory nut tree that procures maximum membership value is chosen as the best compromising solution for the MFED problem.

The flowchart of the FSSA approach to solve EELD problem is shown in Fig. 2.

\section{Numerical results and discussion}

To demonstrate the effectiveness of the proposed FSSA approach, it is applied to a three-area, 10-unit power system for the SAMFED and MAMFED problems. The coefficients of multi-fuel cost and emission, generation limits of the generating units, and tie-line capacity limits are taken from [7].

Table 2 Optimal solution acquired by the proposed approach for fuel cost objective

\begin{tabular}{lll}
\hline Unit & Fuel Types & FSSA \\
\hline 1 & 2 & 218.5032 \\
2 & 1 & 212.3104 \\
3 & 1 & 280.4736 \\
4 & 3 & 239.5643 \\
5 & 1 & 278.5875 \\
6 & 3 & 239.7953 \\
7 & 1 & 288.0741 \\
8 & 3 & 239.8211 \\
9 & 3 & 426.4850 \\
10 & 1 & 276.0857 \\
Minimum cost $(\$ / \mathrm{h})$ & & 623.8613 \\
Emission $(\mathrm{kg} / \mathrm{h})$ & & 6460.9222 \\
\hline
\end{tabular}




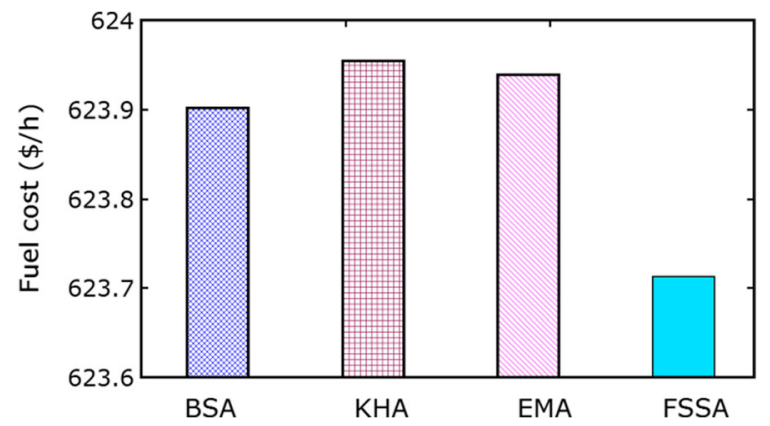

Fig. 3 Performance comparison of the FSSA approach with other techniques for fuel cost objective

The proposed FSSA is executed in Matlab 7.1 on an Intel core i3 processor with 4GB RAM PC, for 50 autonomous trials to assess the solution quality and convergence behavior. The parameter settings of the proposed FBSSA are presented in Table 1.

Three scenarios are researched and the results of the proposed FSSA are compared with Krill Herd Algorithm (KHA), Exchange Market Algorithm (EMA) and other state-of-art heuristic approaches.

Scenario 1. Minimization of the total fuel cost and total pollutant emission individually in the SAMFED problem.

Scenario 2. Minimization of both the total fuel cost and the total emission level simultaneously in the SAMFED problem.

Scenario 3. Minimization of the total fuel cost in the MAMFED problem.

\subsection{Scenario 1}

Table 2 presents the optimal generation schedule acquired by the fuel cost minimization of the SAMFED

Table 3 Optimal solution acquired by the FSSA approach for emission objective

\begin{tabular}{lll}
\hline Unit & Fuel Types & FSSA \\
\hline 1 & 2 & 195.6476 \\
2 & 1 & 209.3824 \\
3 & 1 & 299.5748 \\
4 & 3 & 256.2852 \\
5 & 1 & 290.5376 \\
6 & 1 & 168.3943 \\
7 & 2 & 364.2906 \\
8 & 3 & 260.6658 \\
9 & 3 & 439.9363 \\
10 & 1 & 215.2854 \\
Fuel cost $(\$ / \mathrm{h})$ & & 668.6195 \\
Minimum Emission $(\mathrm{kg} / \mathrm{h})$ & & 6044.4789 \\
\hline
\end{tabular}

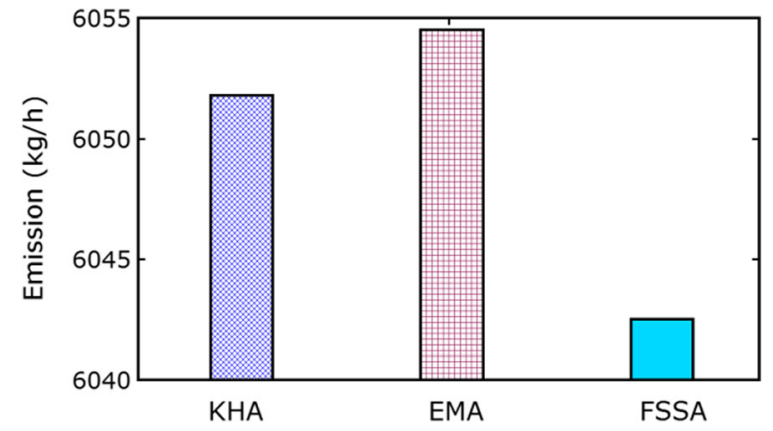

Fig. 4 Performance comparison of the FSSA approach with other techniques for emission objective

using the proposed FSSA approach. The proposed approach is compared with the known approaches such as EMA, KHA, and BSA [20] in Fig. 3. As can be seen from Fig. 3, the FSSA approach outflanks the aforementioned approaches with the lowest total fuel cost.

The optimal values of generating units obtained by the FSSA for emission minimization are given in Table 3, with the minimum emission value of $6042.5066 \mathrm{~kg} / \mathrm{h}$. Figure 4 compares the results from different approaches, which shows that the FSSA approach reduces emission level by $9.31 \mathrm{~kg} / \mathrm{h}$ and $12.07 \mathrm{~kg} / \mathrm{h}$ compared to the KHA and EMA approaches, respectively.

\subsection{Scenario 2}

For this scenario, the total fuel cost and emission of the SAMFED are simultaneously minimized, so the objective function of the EED given in (3) is minimized. In order to provide the best trade-off, the weights $\mathrm{w}_{1}$ and $\mathrm{w}_{2}$ are varied in the range of 0 to 1 in step of 0.1 so that their sum is one. The non-dominated solution sets acquired by the FSSA approach are depicted in Table 4, and the solution corresponding to the maximum membership value estimation is chosen as the BCS. It is seen from

Table 4 Non-dominated solutions for various weighting values acquired by the proposed FSSA approach

\begin{tabular}{lllll}
\hline $\boldsymbol{W}_{\mathbf{1}}$ & $\boldsymbol{W}_{\mathbf{2}}$ & Fuel cost $(\mathbf{\$} / \mathbf{h})$ & Emission $(\mathrm{kg} / \mathrm{h})$ & Membership value $\left(\boldsymbol{\mu}_{\boldsymbol{D}}\right)$ \\
\hline 1 & 0 & 623.7129 & 6469.4021 & 0.080396 \\
0.9 & 0.1 & 628.4644 & 6394.3036 & 0.086413 \\
0.8 & 0.2 & 632.7907 & 6321.5892 & 0.092708 \\
0.7 & 0.3 & 637.5308 & 6255.6224 & 0.097025 \\
$\mathbf{0 . 6}$ & $\mathbf{0 . 4}$ & $\mathbf{6 4 1 . 9 4 7 4}$ & $\mathbf{6 1 9 5 . 3 6 1 3}$ & $\mathbf{0 . 1 0 0 8 2 0}$ \\
0.5 & 0.5 & 644.7538 & 6174.6632 & 0.099919 \\
0.4 & 0.6 & 649.7948 & 6149.9250 & 0.095957 \\
0.3 & 0.7 & 654.8735 & 6121.4394 & 0.092635 \\
0.2 & 0.8 & 659.9145 & 6095.9525 & 0.088814 \\
0.1 & 0.9 & 664.9179 & 6071.2147 & 0.084916 \\
0 & 1 & 670.7220 & 6042.5066 & 0.080396 \\
\hline
\end{tabular}


Table 5 BCS acquired by the FSSA approach

\begin{tabular}{lll}
\hline Unit & Fuel Types & FSSA \\
\hline 1 & 2 & 204.1769 \\
2 & 1 & 213.7536 \\
3 & 1 & 297.1947 \\
4 & 3 & 254.9654 \\
5 & 1 & 288.2184 \\
6 & 1 & 189.5552 \\
7 & 2 & 308.8693 \\
8 & 3 & 236.4719 \\
9 & 3 & 423.2964 \\
10 & 1 & 283.4982 \\
Fuel cost $(\$ / \mathrm{h})$ & & 641.9474 \\
Emission $(\mathrm{kg} / \mathrm{h})$ & & 6195.3613 \\
\hline
\end{tabular}

Table 4 that the BCS are determined at $w_{1}=0.6$ and $w_{2}=0.4$ corresponding to $\mu_{\mathrm{D}}=0.100820$. For the FSSA algorithm, the total fuel cost is 641.9474 and the total pollutant emission is $6195.3613 \mathrm{~kg} / \mathrm{h}$. Table 5 presents the optimal generation schedule of the SAMFED problem by minimizing both the total fuel cost and emission.

The performance indices of the EED problem such as fuel cost performance index (FCPI) and emission cost performance index (ECPI) are further performed. FCPI and ECPI can be expressed as:

$$
\begin{aligned}
& F C P I=\frac{F_{b c s}-F_{\text {min }}}{F_{\text {max }}-F_{\text {min }}} \times 100 \\
& E C P I=\frac{E_{b c s}-E_{\text {min }}}{E_{\text {max }}-E_{\text {min }}} \times 100
\end{aligned}
$$

The performance indices of the FSSA approach are compared with the results obtained using the KHA and EMA approaches, as shown in Fig. 5. It is evident that the performance indices acquired using the FSSA approach are superior to the other approaches, with lower divergence. Figure 6 shows the Pareto optimal fronts (POF) curves of different approaches. It is seen that the POF curve of the FSSA algorithm remains closer to the

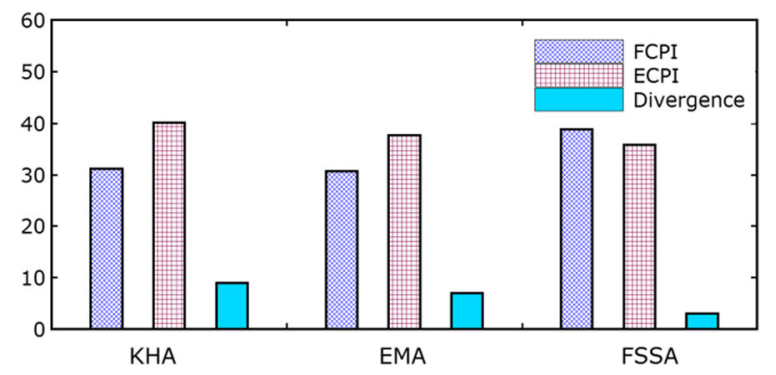

Fig. 5 Performance comparison of the FSSA algorithm with other techniques for EED

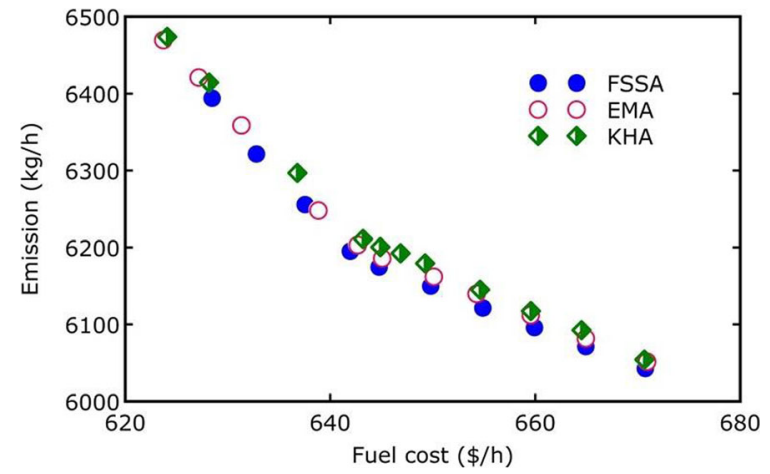

Fig. 6 POF curves obtained from various approaches

axes, which implies that the FSSA approach offers better results than the KHA and EMA approaches.

\subsection{Scenario 3}

In this scenario, fuel cost minimization of MAMFED is addressed. A 3-area, 10-unit test system is tested with transmission losses, and VPL and MFA impacts taken into consideration. Areas 1, 2, and 3 comprise four, three and three generating units, respectively as displayed in Fig. 7. The total power demand of the system is $2700 \mathrm{MW}$, and the power demand shares of areas 1, 2, and 3 are $50 \%, 25 \%$, and $25 \%$ of the total load demand, respectively. The power flow from one area to another is restricted to $100 \mathrm{MW}$. Table 6 presents the simulation results obtained by the proposed SSA approach. It can be seen that the optimal generation cost obtained by the SSA approach is $654.4665 \$ / \mathrm{h}$, which is the lowest among all the approaches. Area 1 imports power from areas 2 and 3, while area 3 also exports power to area 2 .

The results of the SSA approach are compared with those of RCGA [14], ABC [14], TLBO [15], EPSO [24],

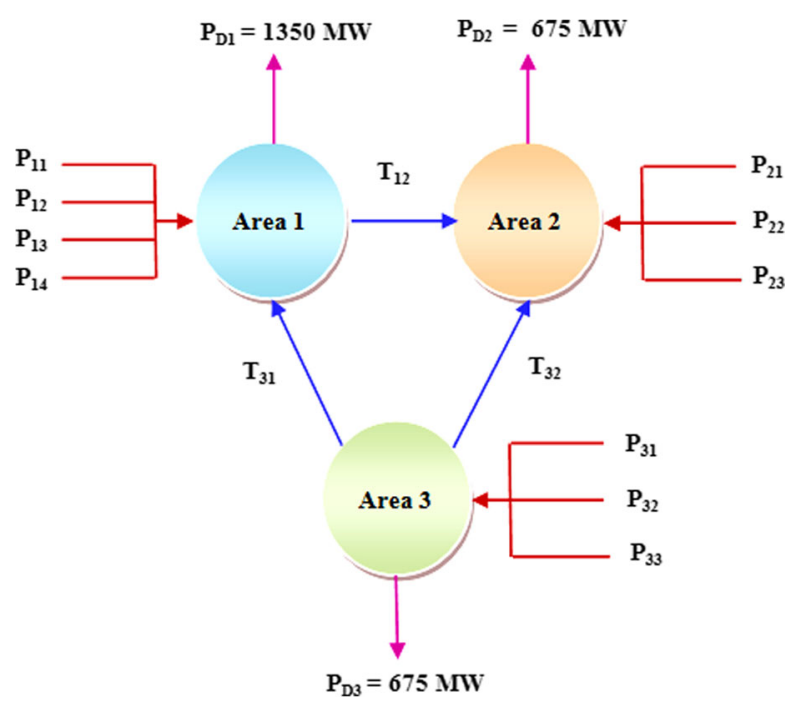

Fig. 7 Schematic diagram of the three-area system 
Table 6 Best dispatch solution incurred by the proposed SSA algorithm for MAMFED problem

\begin{tabular}{|c|c|c|c|c|}
\hline Unit & $P_{i j, \text { min }}(M W)$ & $P_{i j, \max }(M W)$ & Fuel types & Power generation (MW) \\
\hline$P_{1,1}$ & 196 & 250 & 2 & 225.7694 \\
\hline$P_{1,2}$ & 157 & 230 & 1 & 211.5842 \\
\hline$P_{1,3}$ & 388 & 500 & 2 & 491.3265 \\
\hline$P_{1,4}$ & 200 & 265 & 3 & 238.5371 \\
\hline$P_{2,1}$ & 190 & 338 & 1 & 252.6869 \\
\hline$P_{2,2}$ & 200 & 265 & 3 & 235.7538 \\
\hline$P_{2,3}$ & 200 & 331 & 1 & 264.7952 \\
\hline$P_{3,1}$ & 200 & 265 & 3 & 236.4286 \\
\hline$P_{3,2}$ & 213 & 370 & 1 & 330.8961 \\
\hline$P_{3,3}$ & 200 & 362 & 1 & 247.9518 \\
\hline$T_{21}$ & 99.9792 & $P_{\mathrm{L} 1}$ & 17.2813 & \\
\hline$T_{31}$ & 100.0848 & $P_{\mathrm{L} 2}$ & 9.8161 & \\
\hline$T_{32}$ & 31.5594 & $P_{L 3}$ & 8.6328 & \\
\hline \multicolumn{3}{|c|}{ Fuel cost $(\$ / h)$} & 653.8601 & \\
\hline
\end{tabular}

EMA and CFLBO approaches in Fig. 8, and it shows that the proposed strategy outperforms the others with regard to finding the best generation schedule.

\subsection{Statistical analysis of the FSSA algorithm \\ 5.4.1 Multi-objective performance indicators}

The multi-objective performance indicators such as generational distance (GD), spacing metric (SM) and ratio of non-dominated individuals (RNI) are used to examine the performance of the proposed approach for Scenario 3.

The smaller estimations of GD and SM demonstrate better union to the POF curve, and better conveyance and assorted variety of the non-dominated solutions, respectively. A closer estimation of one for RNI index demonstrates that the greatest number of arrangements in a populace are non-dominated. The GD, SM, and RNI measures obtained by the proposed FSSA approach in 50 autonomous trials are shown as box and whisker plots in Fig. 9, which indicate that the FSSA algorithm

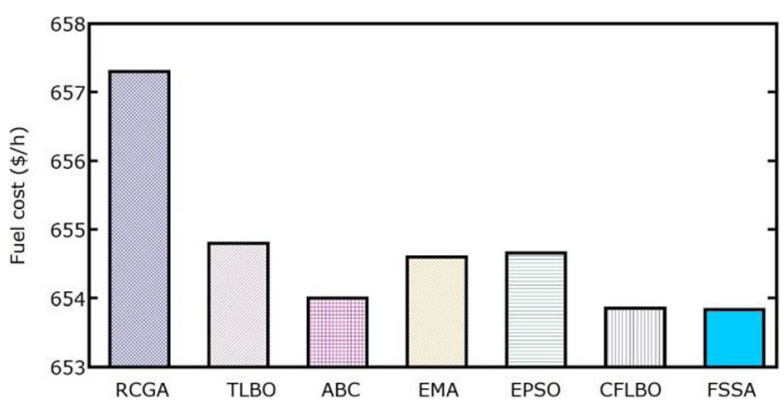

Fig. 8 Comparison of generation costs incurred by various approaches for scenario3 has estimated the smaller values regarding the GD and $\mathrm{SM}$ indices, and closest to one for the RNI indicator.

\subsubsection{Fuel cost improvement percentage}

Fuel cost improvement percentage (IP) is the ratio of fuel cost difference obtained between two approaches to the higher value of fuel cost obtained, expressed as a percentage as:

$I P=\frac{\text { fuel cost of the compared approach }- \text { fuel cost of the suggested approach }}{\text { fuel cost of the compared approach }} \times 100$

The IPs obtained by the FSSA approach and the existing heuristic approaches for scenario 3 are compared in Fig. 10, ranging from $0.113 \%$ to $0.5234 \%$. It is noteworthy that the IP of the FSSA approach is high for MAMFED with VPL impacts. Thus, it can conclude that the proposed FSSA approach provides better results than the other approaches.

\subsubsection{Computational efficiency}

Tables 2, 5, and 6 show that the minimum fuel costs achieved by the FSSA approach are 623.7129, 641.9474, and $654.4665 \$ / \mathrm{h}$ for scenarios 1,2 , and 3 , respectively. These costs are lower than the ones presented in recent literature. Figure 11 shows the number of function evaluation adopted by the FSSA and EMA strategies for the various scenarios, indicating lower number of function evaluation with FSSA. Thus, the FSSA technique is more computationally effective than the EMA strategy. 

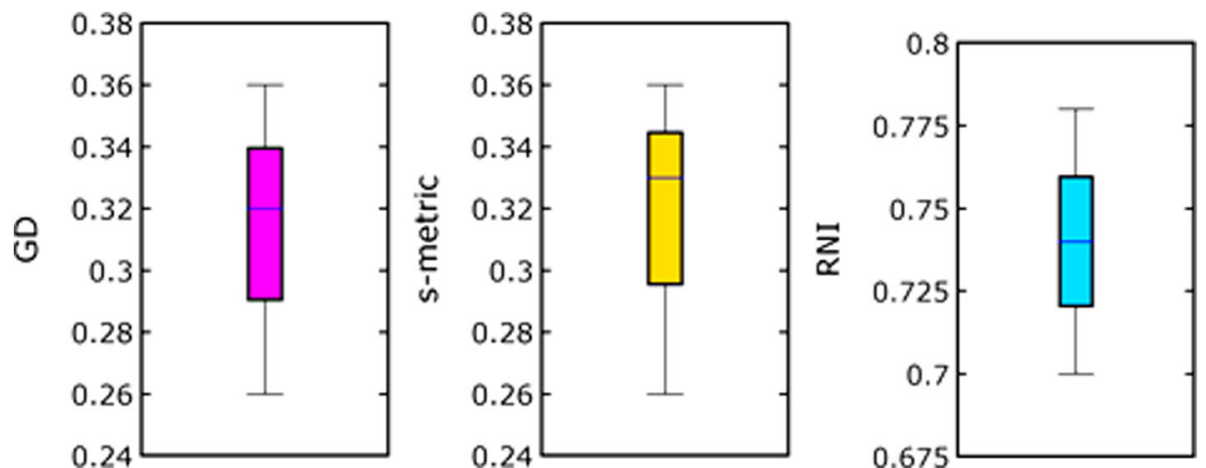

Fig. 9 Boxplots of multi-objective performance measures

\subsubsection{Convergence graph}

The convergences of the EMA and FSSA approaches are compared in Fig. 12. As shown, the FSSA approach requires lower number of iterations to converge to the globally optimal solution.

\section{Conclusion}

The MAMFED problem, as an imperative issue in modern power system analysis, is addressed in this paper. The optimal generation schedule of all committed units and power transfer between the areas through tie-lines are determined. The FSSA approach is used to solve the SAMFED and the MAMFED problems, while the transmission line losses, VPL impacts, and tie-line limits of the power system are addressed. The bi-objective function is transferred into a single objective function by weighted sum approach. Moreover, a fuzzy decision strategy is introduced to find one of the Pareto optimal fronts as the best compromised solution. To demonstrate the effectiveness of the FSSA approach in terms of solution quality, computational efficiency and convergence speed, it is used to solve the MFED problems with three different scenarios and the results are compared with other existing approaches. The results prove that the proposed FSSA approach is an

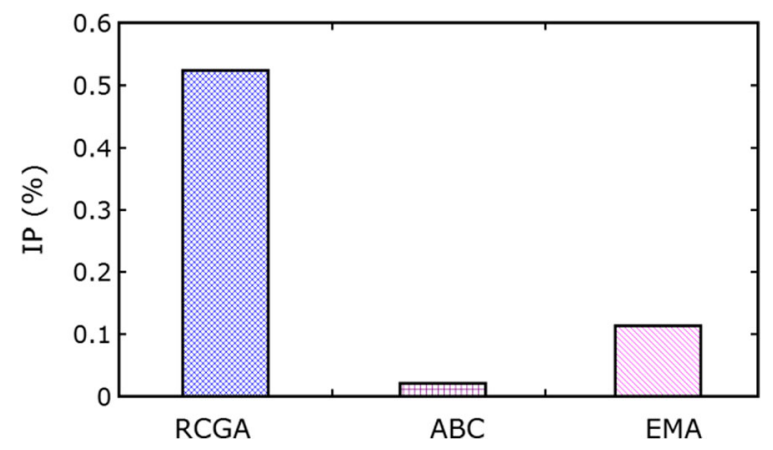

Fig. 10 Comparison of IPs obtained by various approaches for scenario 3 efficient method for solving the MFMAED problem and can provide better compromised solution than the other approaches. This research work not only offers an advanced optimizer for the MFMAED problem but also advances the use of the evolutionary approaches in the energy optimization domain. For future work, it will be intriguing to implement this compelling approach to solve other economic operation problems of hybrid wind-solar-thermal power systems.

\section{Nomenclature}

$a_{i}, b_{i}, c_{i}$ cost coefficients of generator $i$

$a_{i j}$, $b_{i j}$, $c_{i j}$ cost coefficients of generator $i$ in area $j$

$C_{D}$ drag coefficient

$C_{L}$ lift coefficient

$d_{i}, e_{i}$ cost coefficients of the VPL effect of generator $i$

$d_{g}$ gliding distance

$D$ drag force

$e_{i j}$, $f_{i j}$ cost coefficients of the VPL effect of generator $i$ in area $j$

$E_{i}$ emission of the generator $i$

$F_{i}$ fuel cost of the generator $i$

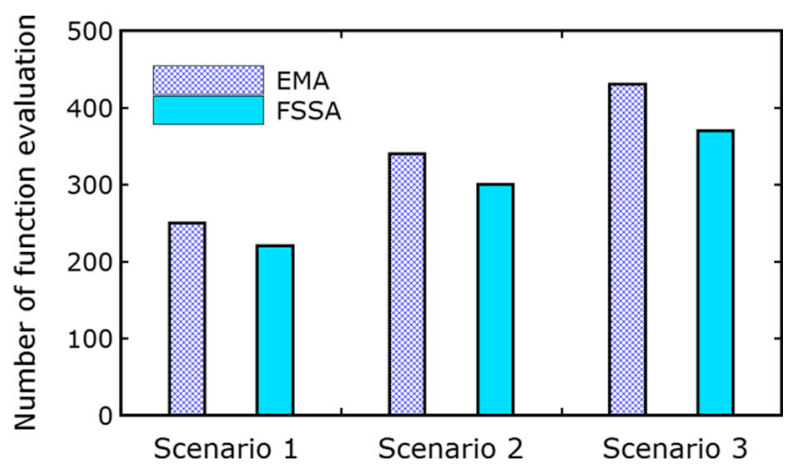

Fig. 11 Comparison of function evaluation adopted by various scenarios 


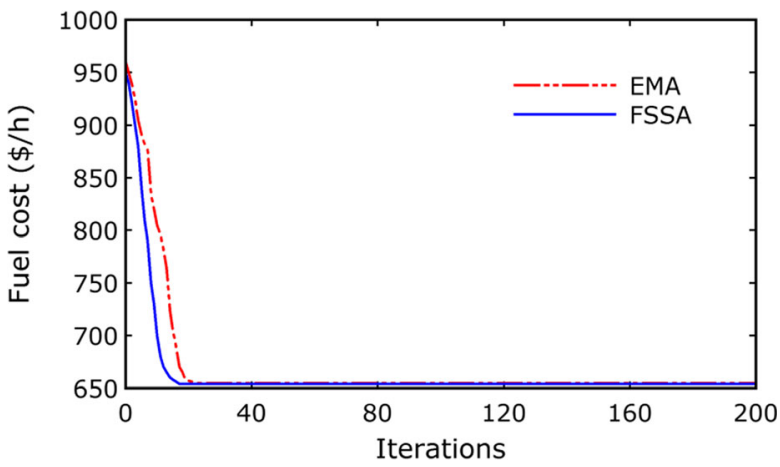

Fig. 12 Convergence comparison of the FSSA and EMA approaches

$F_{i j}\left(P_{i j}\right)$ fuel cost of the generator $i$ in area $j$ $F_{j}^{\text {max }} \mathrm{F}_{j}^{\max }$ and $F_{j}^{\text {min }} \mathrm{F}_{j}^{\text {min }}$ maximum and minimum values of $j$ th objective function respectively $F_{\mathrm{bcs}}$ and $E_{\mathrm{bcs}}$ fuel cost and emission attained by EED $F_{\min }$ and $E_{\max }$ fuel cost and emission attained by ED minimization respectively

$F_{\max }$ and $E_{\min }$ fuel cost and emission attained by EmD minimization respectively

$G_{c}$ Gliding constant

$h$ price penalty factor in $\$ / \mathrm{h}$

$h_{g}$ gliding height

IP improvement percentage

$k$ number of fuel alternatives

$L$ lift force

$M_{i}$ number of participated generators in area $i$

$M$ number of non-dominated solutions

$n$ maximum number of objects

$n g$ total number of generating units

$P_{D}$ power demand

$P_{i j}$ Real power generation of generator $j$ in area $i$

$P_{\dot{v} \text { min }} P_{\dot{v} \text { max }}$ minimum and maximum generation of unit $i$

$P_{i p \text { min }} P_{i j \text { max }}$ Minimum and maximum generation $j$ in area $i$

$P_{d p}$ predator presence probability

$r_{a}$ and $r_{b}$ randomly distributed numbers in $[0,1]$

$r_{1}, r_{2}$ and $r_{3}$ random numbers in the range of $[0,1]$

$S$ surface area of body

$S_{\min }$ minimum value of seasonal constant

$t$ current iteration

$t_{\text {max }}$ maximum iteration value

$T_{i z}$ tie line power stream from area $i$ to area $z$

$T_{i z \text {, max }}$ maximum tie line power stream from area $i$ to area $z$

$-T_{i z \text {, max }}$ Maximum tie line power stream from area $z$ to area $i$

$V$ speed

$w$ weight or compromise factor
$X_{h}$ position of squirrel individual which reached the hickory tree

$X_{L}, X_{U}$ lower and upper bounds of squirrel individual

$x_{j}^{\text {min }}$ and $x_{j}^{\text {max }}$ lower and upper limits of variable $j$

tan ( ) gliding angle

$\beta$ constant

$\rho$ density of air emission coefficients of generator $i$

\section{Acknowledgements}

Authors would like to thank Government College of Engineering Dharmapuri and Annamalai University Chidambaram for providing necessary research facilities.

Authors' contributions

VPS as the corresponding author, contributed significantly to the research, writing and submission of the paper. PDS provided decisive comments and technical supervision for the algorithm. All the authors read and approved the submitted manuscript.

Funding

No funding received from any agency.

Availability of data and materials

No data has been taken from any sources.

\section{Declarations}

Competing interests

The authors declare that they have no competing interests.

\section{Author details}

${ }^{1}$ Department of Electrical and Electronics Engineering, Government College of Engineering, Dharmapuri, Tamilnadu 636 704, India. ${ }^{2}$ Department of Electronics and Communication Engineering, FEAT, Annamalai University, Chidambaram, Tamilnadu 608 002, India.

Received: 1 June 2020 Accepted: 22 February 2021

Published online: 02 April 2021

\section{References}

1. Shoults, R. R., Chang, S. K., Helmick, S., \& Grady, W. M. (1980). A practical approach to unit commitment, economic dispatch and savings allocation for multiple-area pool operation with import/export constraints. IEEE Transactions on Power Apparatus and Systems, PAS-99(2), 625-635 https:// doi.org/10.1109/TPAS.1980.319654.

2. Quintana, V. H., Lopez, R., Romano, R., \& Valadez, V. (1981). Constrained economic dispatch of multi-area systems using the dantzig-wolfe decomposition principle. IEEE Transactions on Power Apparatus and Systems, PAS-100(4), 2127-2137 https://doi.org/10.1109/TPAS.1981.316490.

3. Ouyang, Z., \& Shahidehpour, S. M. (1991). Heuristic multi-area unit commitment with economic dispatch. IEE Proceedings C - Generation Transmission and Distribution, 242-252 https://doi.org/10.1049/ip-c.1991. 0030

4. Wang, C., \& Shahidehpour, S. M. (1992). A decomposition approach to nonlinear multi-area generation scheduling with tie-line constraints using expert systems. IEEE Transactions on Power Systems, 7(4), 1409-1418 https:// doi.org/10.1109/59.207362.

5. Streiffert, D. (1995). Multi-area economic dispatch with tie line constraints. IEEE Transactions on Power Systems, 10(4), 1946-1951 https://doi.org/10.11 09/59.476062.

6. Yalcinoz, T., \& Short, M. J. (1988). Neural networks approach for solving economic dispatch problem with transmission capacity constraints. IEEE Transactions on Power Systems, 13(2), 307-313 https://doi.org/10.1109/59. 667341.

7. Thang, N. T. (2013). Economic emission load dispatch with multiple fuel options using hopfield Lagrange network. International Journal of Advanced Science and Technology, 57, 9-24 https://doi.org/10.14257/ijhit.2015.8.1.27. 
8. Kuo, C. C. (2008). A novel string structure for economic dispatch problems with practical constraints. Energy Conversion and Management, 49, 35713577 https://doi.org/10.1016/j.enconman.2008.07.007.

9. Li, Y. Z., Jiang, L., Wu, Q. H., et al. (2017). Wind-thermal power system dispatch using MLSAD model and GSOICLW algorithm. Knowledge Based Systems, 116, 94-101 https://doi.org/10.1016/j.knosys.2016.10.028.

10. Jayabarathi, T., Sadasivam, G., \& Ramachandran, V. (2000). Evolutionary programming based multi-area economic dispatch with tie line constraints, Electric Machines and Power Systems, 28(12), 1165-1176 https://doi.org/10.1 080/073135600449044.

11. Manoharan, P. S., Kannan, P. S., Baskar, S., \& Iruthayarajan, M. (2009). Evolutionary algorithm solution and KKT based optimality verification to multi-area economic dispatch. International Journal of Electrical Power and Energy Systems, 31(7-8), 365-373 https://doi.org/10.1016/j.jjepes.2009.03.010.

12. Sharma, M., Manjaree, P., \& Laxmi, S. (2011). Reserve constrained multi-area economic dispatch employing differential evolution with time varying mutation. International Journal of Electrical Power and Energy Systems, 33(3), 753-766 https://doi.org/10.1016/j.ijepes.2010.12.033.

13. Somasundaram, P., \& Jothi Swaroopan, N. M. (2011). Fuzzified particle swarm optimization algorithm for multi-area security constrained economic dispatch. Electric Power Components and Systems, 39(10), 979-990 https:// www.researchgate.net/deref/http\%3A\%2F\%2Fdx.doi.org\%2F10.1080\%2F1532 5008.2011.552094.

14. Basu, M. (2013). Artificial bee colony optimization for multi-area economic dispatch. International Journal of Electrical Power and Energy Systems, 49(7), 181-187 https://doi.org/10.1016/j.ijepes.2013.01.004.

15. Basu, M. (2014). Teaching-learning-based optimization algorithm for multiarea economic dispatch. Energy, 68(4), 21-28 https://doi.org/10.1016/j. energy.2014.02.064.

16. Basu, M. (2017). Fast convergence evolutionary programming for multi-area economic dispatch. Electric Power Components and Systems, 45(15), 16291637 https://doi.org/10.1080/15325008.2017.1376234

17. Nguyen, K. P., Dinh, N. D., \& Fujita, G. (2015). Multi-area economic dispatch using hybrid cuckoo search algorithm. In Proc. 50th International Universities Power Engineering Conference, stoke on Trent, UK, (pp. 1-4) https://doi.org/1 $0.1109 /$ UPEC.2015.7339777.

18. Ghasemi, M., Aghaei, J., Akbari, E., et al. (2016). A differential evolution particle swarm optimizer for various types of multi-area economic dispatch problems. Energy, 107(7), 182-195 https://doi.org/10.1016/j.energy.2016.04. 002.

19. Zhang, P., Ma, W., \& Dong, Y. (2019). Multi-area economic dispatching using improved grasshopper optimization algorithm. Evolving Systems. https://doi. org/10.1007/s12530-019-09320-6

20. Modiri-Delshad, M., Aghay Kaboli, S. H., \& Taslimi-Renani, E. (2016). Backtracking search algorithm for solving economic dispatch problems with valve-point effects and multiple fuel options. Energy, 116(1), 637-649 https:// doi.org/10.1016/j.energy.2016.09.140.

21. Mokarram, M. J., Niknam, T., Aghaei, J., et al. (2019). Hybrid optimization algorithm to solve the nonconvex multiarea economic dispatch problem IEEE Systems Journal, 13(3), 3400-3409 https://doi.org/10.1109/JSYST.2018.2 889988.

22. Mokarram, M. J., Gitizadeh, M., Niknam, T., \& Niknam, S. (2019). Robust and effective parallel process to coordinate multi-area economic dispatch (MAED) problems in the presence of uncertainty. IET Generation, Transmission and Distribution, 13(18), 4197-4205 https://doi.org/10.1049/ietgtd.2019.0319.

23. Qin, J., Wan, Y., Yu, X., \& Kang, Y. (2020). A newton method-based distributed algorithm for multi-area economic dispatch. IEEE Transactions on Power Systems, 35(2), 986-996 https://doi.org/10.1109/TPWRS.2019.2943344.

24. Mohammadian, M., Lorestani, A., \& Ardehali, M. M. (2018). Optimization of single and multi-areas economic dispatch problems based on evolutionary particle swarm optimization algorithm. Energy, 116, 710-724. https://doi. org/10.1016/j.energy.2018.07.167.

25. Suman, M., \& Sakthivel, V. P. (2020). Coulomb's and Franklin's laws based optimization for nonconvex economic and emission dispatch problems. International Energy Journal, 20, 225-238 http://www.rericjournal.ait.ac.th/ index.php/reric/article/view/2273.

26. Jain, M., Singh, V., \& Rani, A. (2019). A novel nature-inspired algorithm for optimization: Squirrel search algorithm. Swarm and Evolutionary Computation, 44(2), 148-175 https://doi.org/10.1016/j.swevo.2018.02.013.

\section{Submit your manuscript to a SpringerOpen ${ }^{\circ}$ journal and benefit from:}

- Convenient online submission

- Rigorous peer review

- Open access: articles freely available online

- High visibility within the field

- Retaining the copyright to your article

Submit your next manuscript at $\boldsymbol{\nabla}$ springeropen.com 